\title{
Lead exposure and early child neurodevelopment among children 12-24 months in Kinshasa, the Democratic Republic of Congo
}

\author{
Espérance Kashala-Abotnes ${ }^{2} \cdot$ Pépé Penghele Mumbere $^{1}$. \\ Jeannette Mukanya Mishika ${ }^{1}$ - Ally Omba Ndjukendi ${ }^{3}$ - Davin Beya Mpaka ${ }^{3}$. \\ Makila-Mabe Guy Bumoko ${ }^{1}$. Tharcisse Kalula Kayembe ${ }^{1}$. \\ Désiré Tshala-Katumbay ${ }^{1,4}$. Théodore Kayembe Kazadi ${ }^{1}$. \\ Daniel Luwa E-Andjafono Okitundu ${ }^{1}$
}

Received: 2 October 2015 / Accepted: 25 April 2016 / Published online: 10 May 2016 (C) The Author(s) 2016. This article is published with open access at Springerlink.com

\begin{abstract}
Childhood lead exposure remains a problem in developing countries, and little is known about its effects on early child neurodevelopment and temperament in the Democratic Republic of Congo (DRC). We, therefore, conducted this study to determine the association between lead exposure and the neurodevelopment and behaviour of children aged 12-24 months in Kinshasa, DRC. A crosssectional study was conducted between February and June 2012, and parents of 104 children were invited to participate. Blood lead levels (BLLs) of each child were tested using the flame atomic spectrophotometry method. All children were subject to a clinical examination and assessed with two selected early child neurodevelopmental tools, the Gensini-Gavito and the baby characteristics questionnaire, to measure their neurodevelopment and temperament. Detectable BLLs ranged from 1 to $30 \mu \mathrm{g} / \mathrm{dl}$ with a geometric mean of $6.9(\mathrm{SD} 4.8) \mu \mathrm{g} / \mathrm{dl}$. BLLs at $5-9$ and $\geq 10 \mu \mathrm{g} /$ $\mathrm{dl}$ were significantly associated with the child temperament
\end{abstract}

E. Kashala-Abotnes and P. P. Mumbere contributed equally.

Espérance Kashala-Abotnes

esperance.abotnes@uib.no; esperance_kashala@yahoo.com

1 Department of Neurology, Centre

for Neuro-Psycho-Pathology, University of Kinshasa, Kinshasa, Democratic Republic of Congo

2 Centre for International Health, Department of Global Public Health and Primary Care, University of Bergen, Bergen, Norway

3 Department of Psychiatry, Centre for Neuro-Psycho-Pathology, University of Kinshasa, Kinshasa, Democratic Republic of Congo

4 Centre for Research on Occupational and Environmental Toxicology, Oregon Health and Science University, Portland, OR, USA $(p<0.05)$. Perinatal and maternal factors did not seem to affect early child neurodevelopment and temperament. Children exposed to lead were reported with more temperament difficulties at even blood lead levels $<10 \mu \mathrm{g} / \mathrm{dl}$, suggesting the need for preventive and intervention measures to reduce lead exposure among children in Kinshasa, DRC.

Keywords Childhood · Lead exposure ·

Neurodevelopment $\cdot$ Temperament $\cdot$ Kinshasa/DRC

\section{Background}

Lead exposure remains an important public health challenge in the world, particularly in Africa due to, among other factors, poverty, low level of awareness and poor capacity to enforce legislation [1]. Lead exposure in early childhood have been associated with cognitive deficits, poor school performance and behavioural problems [2].

An increasing number of studies suggested that childhood lead exposure increases the risk for neurodevelopmental delays, behavioural problems and cognitive impairments even at blood lead levels below $10 \mu \mathrm{g} / \mathrm{dl}$ [3-5]. These impairments may persist into adolescence and adulthood, altering the optimal development of exposed children [6, 7].

Recently, the centers for disease control (CDC) eliminated the terminology "level of concern" and advised that children with blood lead levels requiring public health action should be identified using the reference value of $5 \mu \mathrm{g} / \mathrm{dl}$. This value, generated by the national health and nutrition examination survey (NHANES), is based on the 97.5th percentile of blood lead level distribution among children aged 1-5 years old living in the United States of America (http://www.cdc.gov/mmwr/preview/mmwrhtml/ mm6120a6.htm). 
In the Democratic Republic of Congo (DRC), little is known about the effects of lead exposure on the neurodevelopment and behaviour of children in Kinshasa, and so far no studies have investigated such relation in the country. Findings from a recent study conducted in Kinshasa, suggested that a large proportion of children under 6 years of age were exposed to lead with a median blood lead levels of $11.5 \mu \mathrm{g} / \mathrm{dl}$ [8]. The authors reported that the use of leaded gasoline and activities of car battery recycling, such as handmade cooking utensils, appear to constitute the main source of lead exposure in the city of Kinshasa. The traditional use of fired clay for the treatment of gastritis by pregnant women was another significant contributor for elevated blood lead levels among children [8]. Although they highlighted the significant success achieved by the public health system with the removal of lead from gasoline since 2009 , they emphasised the need for further research on the impact of childhood lead exposure [6,9].

To the best of our knowledge, no studies have investigated the impact of childhood lead exposure in Kinshasa, and there is no established level for intervention with regard to lead exposure in DRC. There is, therefore, a need to study the adverse effects of lead exposure on early child neurodevelopment and behaviour in Kinshasa, DRC and to bring awareness among the population as a preventive measure. Our main objective was, therefore, to determine the association between lead exposure and neurodevelopment and behaviour of children aged 12-24 months living in Kinshasa, DRC. The specific objectives were to explore temperament and neurodevelopment of lead exposed children, and contributing factors such maternal psycho-affectivity and child early cerebral morbidity.

\section{Methods}

\section{Participants and design}

A cross-sectional study was conducted at one referral mother and child health care center in Kinshasa, DRC between February and June 2012. The center offers mother and child care and is located in one of the most vulnerable township of Kinshasa. Using convenience sampling, the parents/caregivers of children aged 12-24 months attending mother and child health care center were invited to participate in the study. Signed informed consent was obtained from parents/caregivers for each child.

\section{Exclusion criteria}

All children were examined by a physician. Those with medical history and/or clinical diagnosis of neurological condition such cerebral palsy or epilepsy, as well as children identified with clinical signs of undernutrition (marasmus and kwashiorkor) during the clinical examination and anthropometric measurements were excluded from the study.

\section{Measures and data collection}

A structured interview was conducted with parents/caregivers to identify socio-demographic characteristics, usage of cooking utensils made with recycled lead based material, child birth and health history, maternal psycho-affectivity, factors of cerebral early morbidity, and neurobehavioural development of the child. Parental level of education was used as a proxy for socioeconomic status in this study. This was justified by the difficulty of assessing parental income, which may not objectively reflect the real socioeconomic situation in this township of Kinshasa.

\section{Questionnaires}

The Gensini-Gavito scale is a neurodevelopmental scale used to evaluate the psychomotor development of children in three domains, which are motor functions, communication/language and social adaptation [10]. The scale generates a psychomotor developmental quotient (PDQ) expressed in percentage. The PDQ is the ratio between the child real age (RA) obtained from his acquisition in all the three domains at the time of the examination and his chronological age $(\mathrm{CA})$ times 100 : $\mathrm{PDQ}=(\mathrm{RA} / \mathrm{CA} \times 100)$. The scale is widely used in paediatric clinic in Kinshasa and has been locally validated at Kinshasa University Hospital [11]. The PDQ reported was used as continuous variable in the analysis.

The baby characteristics questionnaire (BCQ) is a brief 7-items questionnaire, based on the infant characteristics questionnaire (ICQ) developed by Bates et al. in 1979 [12]. The questionnaire invites parents/caregivers to indicate how they perceived the child temperament, focusing on difficult temperament, prone to irritability, agitation and negative response to restraints for each question on a scale from 1 (easy) to 7 (difficult). A total score is calculated by adding the scores of each question. A score below 35 indicates a perception as a child with an easy temperament, while a score $\geq 35$ denotes a perception as a child with a difficult temperament. The scores reported were analysed as continuous variable in the analysis to compare children temperament between BLLs group's categories.

\section{Blood lead levels}

Blood lead levels (BLLs) were tested using the flame atomic spectrophotometry method at the Research Centre for Geology and Mining in Kinshasa/DRC. The Centre 
participates in quality insurance programs from the British Geological Survey and the Korea Institute of Geosciences and Minerals Resources. The Venous blood was collected by venipuncture in an EDTA sealed tube and kept at low temperature $\left(T 4{ }^{\circ} \mathrm{C}\right)$ and send for analysis at the Research Centre for Geology and Mining in Kinshasa/DRC. Samples were carefully handled to avoid contamination during the process of collection, transport and analysis. Control of contamination is done in relation to the lead standard solution (étalon) and the lab working environment, which does not allow external contamination by lead. Samples are treated in closed bombshell with inorganic acids in secured and controlled lab environment. BLLs were measured using the flame atomic absorption spectrophotometry with a detection range of $0.1 \mu \mathrm{g} / \mathrm{dl}$ for $5 \mu \mathrm{g}$ of aqueous solution by the technique of extraction and prior concentration of the sample. The reference value of BLLs $\geq 5 \mu \mathrm{g} / \mathrm{dl}$ was used in this study as the country does not yet have a reference value for intervention in children. This value is chosen based on the value recommended by the advisory committee on childhood lead poisoning prevention (ACCLPP) of the centers for disease control and prevention (CDC) for children aged $1-5$ years of age.

\section{Usage of lead-containing cooking utensils}

During the structured interview with parents, they were asked whether or not they were using cooking utensils made from recycled car batteries for cooking the child's food. These pots are commonly used in the area and have been identified as one of the common source of lead exposure in Kinshasa [6].

\section{Clinical examination}

Dr. Mumbere saw all children and performed their clinical examination. He was blinded to their BLLs.

\section{Ethical considerations}

Ethical approval was granted by the Institutional Review Board Ethics Commission for Human Research at the University of Kinshasa, DRC. Only children whose parents gave informed consent were invited to participate in the study.

\section{Statistical analysis}

The statistical package for social sciences (SPSS) version 23.0 was used for data analysis. Descriptive analyses were used for sample characteristics. Since BLLs were not normally distributed, non-parametric analyses were used. Mean ranks were compared using Kruskal-Wallis test between groups. Kendall's tau was used as measure of correlations, and generalised linear models (GLM) analyses with Poisson identity link were used to adjust for covariates, and to measure associations between risk factors at different BLLs. All tests were 2-tailed, and results were considered statistically significant when $p$ values were less than or equal to 0.05 .

\section{Results}

\section{Subjects}

Of the 104 children selected to participate in the study, six were excluded due to signs of malnutrition identified during the clinical examination. Four did not have detectable blood lead levels and nine mothers decline to participate. Biological parents, mainly mothers gave their informed consent and were the ones interviewed.

Totally 89 children with measurable BLLs were retained and included in the study.

\section{Socio-demographic characteristics}

The children's age varied between 12 and 24 months with a mean of 17.5 (SD 4.3). The family size ranged from 1 to 6 children per family with a mean of 2.8 (SD 1.7). There were more boys $52(58.4 \%)$ than girls $37(41.6 \%)$, but no gender differences were observed. More than half 48 (54\%) of the children were 18 months old and above. The majority of children $72(81.2 \%)$ had four siblings or less, and a total of sixty-one children $(68.5 \%)$ were the youngest sibling. Seventy-seven $(86.5 \%)$ fathers had a secondary and above level of education, while fewer mothers 66 (74\%) had a secondary and above level of education.

Regarding the context of lead exposure, $92 \%$ reported to have lived in the area for more than 2 years, and $79 \%$ reported using handmade cooking utensils made from recycled battery materials for cooking family and baby food.

\section{Maternal socioemotional characteristics and child medical history}

The mean age of the parents at birth was 29.4 years (SD 6.5) for the mothers and 36.9 years (SD 6.3) for the fathers. There were also a good proportion of mothers reporting psycho-affective symptoms: undesired pregnancies $(68 \%)$ and lack of happiness in the early stage of the pregnancy $(63.5 \%)$. Almost all children $(91 \%)$ were born at term with a mean birthweight of 3291.5 grams (SD 593) and normal delivery. Only $25 \%$ were exclusively breastfed up to 6 months. None were reported with risk factors for early cerebral morbidity. We did not find a statistically significant 
correlation between mother socioemotional characteristics (age at birth, pregnancy, labour, delivery, birthweight) and the child temperament and neurodevelopment as reported with the BCQ and Gensini-Gavito scale (Kendall's correlation $p$ value $>0.05$ ).

\section{Child early neurodevelopment and temperament}

According to the mothers report, the PDQ mean percentage on the Gensini-Gavito scale was 90.68 (SD 14.0) and the mean score of the child temperament on the BCQ was 34.73 (SD 11.7). In general, $52 \%$ of the children were reported with a neurodevelopment corresponding to their age according to the PDQ. Looking into the subscales of the PDQ, only $8 \%$ of the children were reported as delayed on the motor neurodevelopment and $21 \%$ on the communication/language development. More than half (58\%) were reported as delayed on the domain of social adaptation, suggesting social adaptation as the neurodevelopment domain where parents seem to report more difficulties.

On the evaluation of the child temperament as measured with the BCQ using a cut-offs score $\geq 35$, a total of 59 children out of $89(66 \%)$ were reported and perceived as having difficult temperament by parents. This result might be in line with the delays in social adaptation as reported by parents.

\section{Blood lead levels}

Blood lead levels (BLLs) ranged from 1 to $30 \mu \mathrm{g} / \mathrm{dl}$ with a geometric mean of 6.9 (SD 4.8) $\mu \mathrm{g} / \mathrm{dl}$. There was no significant mean difference between boys and girls 7.1 (SD 4.6) vs. 6.4 (SD 5.0), respectively ( $p>0.05$ ).

Using three group categories of BLLs, proportions of children with BLLs between 0 and $4 \mu \mathrm{g} / \mathrm{dl}$, 5-9 and $\geq 10 \mu \mathrm{g} / \mathrm{dl}$ were, respectively $30(34 \%), 36(40 \%)$ and $23(26 \%)$. No gender differences were observed between the three group categories ( $p$ value $>0.05$ ).

\section{Blood lead levels and early child neurodevelopment}

Table 1 summarises the findings of the child neurodevelopment as reported by parents on the Gensini-Gavito scale for BLLs analyses at $0-4,5-9$ and $\geq 10 \mu \mathrm{g} / \mathrm{dl}$.

As shown in the Table 1, no statistical significant differences were observed on the child neurodevelopment between the group categories of BLLs (Kruskal-Wallis $p$ value $>0.05$ ).

Although no statistical significant difference was observed on the three domains of neurodevelopment of the child (motor, language and adaptation), children with BLLs at $5-9 \mu \mathrm{g} / \mathrm{dl}$ were reported with higher mean rank on the
Table 1 Neurodevelopment and behaviour of 12-24 months old children as reported by parents in relation to blood lead levels. Results from a cross-sectional study on lead exposure among 89 children attending a mother and child care center in a vulnerable township of Kinshasa, Democratic Republic of Congo

\begin{tabular}{|c|c|c|c|}
\hline & \multicolumn{3}{|c|}{ Blood lead levels $\mu \mathrm{g} / \mathrm{l}$} \\
\hline & $0-4$ & $5-9$ & $\geq 10$ \\
\hline & $N=30$ & $N=36$ & $N=23$ \\
\hline & Mean rank & Mean rank & Mean rank \\
\hline \multicolumn{4}{|c|}{ Neurodevelopment (gensini-gavito) } \\
\hline $\begin{array}{l}\text { Psychomotor development } \\
\text { quotient }\end{array}$ & 44.98 & 47.08 & 41.76 \\
\hline Motor functions & 45.90 & 43.72 & 45.83 \\
\hline Language and communication & 44.18 & 44.44 & 46.93 \\
\hline Social adaptation & 45.80 & 40.00 & 51.78 \\
\hline \multicolumn{4}{|c|}{ Temperament (baby characteristics questionnaire) } \\
\hline $\begin{array}{l}\text { Total difficult score (difficult } \\
\text { temperament) }\end{array}$ & 38.00 & 44.07 & $55.59^{*}$ \\
\hline Easily irritable & 34.80 & 46.20 & $56.50^{*}$ \\
\hline $\begin{array}{l}\text { Agitation, cries and disruptive } \\
\text { temperament }\end{array}$ & 35.60 & 45.70 & $56.20^{*}$ \\
\hline $\begin{array}{l}\text { Rigorously cries and agitated } \\
\text { when upset }\end{array}$ & 37.10 & 45.50 & $54.50^{*}$ \\
\hline Plays nicely alone & 42.50 & 45.00 & 48.22 \\
\hline
\end{tabular}

$* p$ value $<0.05$ (Kruskal-Wallis test)

social adaptation compared to those with BLLs at $0-4 \mu \mathrm{g} / \mathrm{dl}$ (Kruskal-Wallis $p$ value 0.053 ).

\section{Blood lead levels and child temperament}

BLLs were significantly correlated with the child temperament (Kendall's tau coef. $0.17 ; p$ value 0.02 ). Results from the BCQ on the child temperament as perceived by parents, showed significant differences between the group categories of BLLs at $0-4,5-9$ and $\geq 10 \mu \mathrm{g} / \mathrm{dl}$. Indeed, more children with BLLs $\geq 5 \mu \mathrm{g} / \mathrm{dl}$ were perceived as difficult according to parents compared to those with BLLs between 0 and $4 \mu \mathrm{g} / \mathrm{dl}$ (Pearson Chi square $p$ value 0.002). There was a significant higher proportion of children reported with difficult temperament among those with BLLs $\geq 10 \mu \mathrm{g} / \mathrm{dl}(22 / 23)$, and at 5-9 $\mu \mathrm{g} / \mathrm{dl}$ (22/36), compared to those with BLLs at $0-4 \mu \mathrm{g} / \mathrm{dl}(15 / 30)$ (Pearson Chi square $p$ value 0.002).

In addition, children with BLLs at 5-9 and $\geq 10 \mu \mathrm{g} /$ dl were reported with higher mean rank on the BCQ (Kruskal-Wallis $p$ value $<0.05$ ) (Table 1). 


\section{Independent effects of blood lead levels on child neurodevelopment and temperament}

We looked at the BLLs as a continuous variable, and found a significant correlation with the child temperament (Kendall's tau coef. $0.17 ; p$ value 0.02 ). Similar correlation was observed when both the BLLs and the temperament were analysed as categorical variables for BLLs at, respectively $0-4,5-9$ and $\geq 10 \mu \mathrm{g} / \mathrm{dl}$, and for the difficult temperament at cut-offs $\geq 35$ (Kendall's tau $p$ value 0.001 ).

The independent effects of BLLs on the temperament of the child were analysed using the generalised linear models (GLM) Poisson link Identity analysis to adjust for potential confounders. The factors entered in the model and adjusted for, were risk factors related to the socioeconomic status, the mother and child medical history around pregnancy and birth, and the environmental exposure. This included the following variables: Mother and child antenatal, perinatal and postnatal risk factors (mother affectivity during pregnancy, duration of labour, circumstances of delivery, birthweight, parental age at child birth, morbidity, exclusive breastfeeding etc.), risk factors related to lead exposure and sociodemographic factors (number of years living in the area, daily usage of lead based utensils, family size, rank of the child).

After GLM analyses, a significant association was still observed between BLLs and the temperament of the child ( $p$ value 0.001 ), for both analyses as continuous and categorical variables (BCQ $\geq 35$ and BLLs at $0-4,5-9$ and $\geq 10 \mu \mathrm{g} / \mathrm{dl}$ (coef. 4.3, $p$ value 0.001 ).

\section{Discussion}

The aim of this study was to determine the association between childhood lead exposure and the early child neurodevelopment and temperament among 12-24 month old children living in lead exposed environments in Kinshasa, DRC.

The mean BLLs was $6.9 \mu \mathrm{g} / \mathrm{dl}$, which is considered high and above the recommended level of intervention. Results from this study suggest an association between childhood lead exposure and the child temperament among exposed children in Kinshasa, and support the need for comprehensive neurodevelopmental and behavioural assessments [8]. In addition, our results support and highlight the need for health prevention measures at primary care level among exposed children, as highlighted in previous studies from Kinshasa $[9,13]$.

To summarize, exposed children with BLLs $\geq 5 \mu \mathrm{g} /$ dl significantly scored higher on the BCQ, meaning perceived as child with more difficult temperament. No significant association was found between BLLs and the child neurodevelopment. However, though neurodevelopment was not associated with BLLs, parents of children with BLLs $\geq 5 \mu \mathrm{g} / \mathrm{dl}$ reported more adaptation delays than those of children with BLLs $<5 \mu \mathrm{g} / \mathrm{dl}$. Surprisingly, mother psycho-affectivity during pregnancy did not seem to affect the child neurodevelopment or temperament.

In line with previous studies that have found associations between BLLs and the temperament of the child [4, 14-16], we found that exposed children with higher BLLs were reported to be more prone to irritability, agitation and negative response to restraints. Indeed, parents of children with BLLs at $5-9 \mu \mathrm{g} / \mathrm{dl}$ and $\geq 10 \mu \mathrm{g} / \mathrm{dl}$ reported to have greater difficulties in dealing with the child temperament compared to those at $0-4 \mu \mathrm{g} / \mathrm{dl}$. We found temperamental difficulties at BLLs as early as $5-9 \mu \mathrm{g} / \mathrm{dl}$ as reported in previous studies $[17,18]$, and similar to studies reporting adverse events even at level $<10 \mu \mathrm{g} / \mathrm{dl}[5$, 19]. Based on the presence of adverse effects of childhood lead exposure on child temperament, urgent prevention and intervention measures are needed to reduce lead exposure among children in Kinshasa, DRC, as recommended by earlier studies [20].

Although parents of children with BLLs at 5-9 and at $\geq 10 \mu \mathrm{g} / \mathrm{dl}$ seem to report more delays in the domain of social adaptation, no significant neurodevelopmental difference was observed between children at different categories of BLLs. Our cross sectional design may have prevented us to find an association between level of lead exposure and the early child neurodevelopment. Indeed, child neurodevelopment is a continuous process that requires assessments at different stages of the child development. Longitudinal and follow-up studies would probably be more appropriate to detect early childhood neurodevelopmental delays at different developmental periods. However, delays reported in social adaptation may suggest neurodevelopmental issues that may occur and will be noticeable later in childhood. There is an urgent need to assess and comprehensively explore neurodevelopment of exposed children in Kinshasa, knowing that lead exposed children are at risk for neurodevelopmental delays and intellectual deficits later in their life [21].

Usage of lead-containing handmade kitchenware was the main reported source of exposure in about $80 \%$ of the households. Although we did not explore other sources of exposure among children, the majority of mothers reported a daily usage of lead-containing kitchenware for cooking their baby food. It is well possible that in addition to these utensils, there are other contributing sources of contamination, previously described in Kinshasa, such as fired clay consumed during pregnancies, pesticides and paints [8]. Lead-containing kitchen utensils are commonly used as they are cheap, easily available, and they have been previously identified as one of the main source of lead exposure 
in Kinshasa. Because our results suggest adverse effects of lead exposure on the child temperament, longitudinal studies are needed to better explore the relation between daily lead exposure from handmade kitchen utensils and the child neurobehavioural development.

In contrast to other studies that have reported an association between affective characteristics of the mother and the child temperament $[22,23]$, we were unable to find such an association. Although the majority of mothers reported undesirable pregnancies with lack of happiness in its early stage, we did not use a screening tool to assess maternal anxiety and depressive symptoms during pregnancy, home environment or parenting style. Therefore we could not assume presence of depressive symptoms during pregnancy based on report of unhappiness feelings. It is, therefore, unsure whether or not mothers were depressed or have only reported a transient unhappiness, related to the nondesirability of the pregnancy in its early stage. In addition, only few mothers reported antenatal and perinatal risk factors (during pregnancies and deliveries), and no risk for early cerebral morbidity were identified among the studied children. This could explain why we were unable to find an association between maternal factors during pregnancy and delivery, child's early cerebral morbidities and the child neurodevelopment and temperament. The lack of association between risk factors related to the mother, known as possible confounders, and the early child neurodevelopment and temperament, strengthens the consistency of our results. The association between BLLs and child difficult temperament, as perceived by parents, remained constant even after adjusted analysis and in absence of possible confounders.

This study has some methodological limitations associated with our study design and sample of convenience. Our results cannot be generalised to the whole population of Kinshasa or DRC, though they are of great importance and contribute to the knowledge and awareness of adverse effects of lead exposure on child behavioural development. The study, together with an earlier study conducted in Kinshasa, demonstrate how lead exposure, particularly among children, constitute an important concern for public health [6]. We used a cross-sectional design and tools that did not allow us to assess cognitive functioning of exposed children, which is an important aspect of child neurodevelopment. However, our primary objective was to explore whether there is an association between lead exposure and early child neurodevelopment and temperament. With the selected tools, the GensiniGavito scale and the BCQ, we were able to obtain useful informations on such an association. The assessments were, however, limited to the domains of motor functions, communication, language and social adaptation, and to the child temperament as perceived by parents.
Comprehensive assessments of cognitive, intellectual functioning, school performance of exposed children in the later stage of their development and before their preschool age are needed with different study designs (e.g., longitudinal studies). Another limitation is the limited number of covariates for which the analysis is controlled. We used parental education as a proxy for socioeconomic status (SES) as it is difficult to objectively measure the SES using parental income. This is due to the economic and political instability that have affected the DRC in the past decades, leaving the majority of the population still economically affected though the country is trying to reconstruct its economy.

The strength of the study resides in the awareness brought to better understand adverse effects of lead exposure on early child neurodevelopment and temperament of exposed children in Kinshasa, DRC. To the best of our knowledge, it is the first study investigating such an association among children in DRC. In addition, the study results will benefit policymakers in their formulation of integrated control policy against lead exposure for the protection of child health and their development to their potential of human capital. Dosage of BLLs should be considered among pregnant women consulting at antenatal care clinic and among children presenting with difficult temperament. Because handmade household kitchenware constitutes one of the major sources of lead exposure, it is important as preventive public health measures to inform the population on the associated risk. Preventive and intervention measures on lead exposure will contribute to the optimal wellbeing of exposed children in Kinshasa, DRC.

\section{Conclusions}

Childhood lead exposure affects early child neurodevelopment and temperament of young children in Kinshasa, DRC. Results suggest the need for further investigations to explore the later in-life impact of childhood lead exposure on cognition, intellectual functioning, and school performance of pre- and school aged children.

\section{Compliance with ethical standards}

Competing interests All authors declare that they have no conflict of interests.

Open Access This article is distributed under the terms of the Creative Commons Attribution 4.0 International License (http://creativecommons.org/licenses/by/4.0/), which permits unrestricted use, distribution, and reproduction in any medium, provided you give appropriate credit to the original author(s) and the source, provide a link to the Creative Commons license, and indicate if changes were made. 


\section{References}

1. Mathee A (2014) Towards the prevention of lead exposure in South Africa: contemporary and emerging challenges. Neurotoxicology 45:220-223

2. Liu J et al (2013) Impact of low blood lead concentrations on IQ and school performance in Chinese children. PLoS ONE 8(5):e65230

3. Canfield RL et al (2003) Low-level lead exposure, executive functioning, and learning in early childhood. Child Neuropsychol 9(1):35-53

4. Jusko TA et al (2008) Blood lead concentrations $<10$ microg/dL and child intelligence at 6 years of age. Environ Health Perspect 116(2):243-248

5. Bellinger DC (2008) Very low lead exposures and children's neurodevelopment. Curr Opin Pediatr 20(2):172-177

6. Tuakuila J et al (2013) Blood lead levels in children after phaseout of leaded gasoline in Kinshasa, the capital of Democratic Republic of Congo (DRC). Arch Public Health 71(1):5

7. Wright JP et al (2008) Association of prenatal and childhood blood lead concentrations with criminal arrests in early adulthood. PLoS Med 5(5):e101

8. Tuakuila J et al (2013) Elevated blood lead levels and sources of exposure in the population of Kinshasa, the capital of the Democratic Republic of Congo. J Expo Sci Environ Epidemiol 23(1):81-87

9. Tuakuila $\mathbf{J}$ et al (2012) Worrying exposure to trace elements in the population of Kinshasa, Democratic Republic of Congo (DRC). Int Arch Occup Environ Health 85(8):927-939

10. Gensini H, Gavito MJ (1987)Repères de surveillance du développement de l'enfant en milieu tropical, 170-171, (115-131)

11. Tady MB (2004): Le développement psychomoteur de l'enfant Congolais. Thèse de Doctorat, Faculté de Médecine de l'Université de Kinshasa

12. Bates JE, Freeland CA, Lounsbury ML (1979) Measurement of infant difficultness. Child Dev 50(3):794-803
13. Tuakuila J et al (2014) Toxic and essential elements in children's blood ( $<6$ years) from Kinshasa, DRC (the Democratic Republic of Congo). J Trace Elem Med Biol 28(1):45-49

14. Canfield RL, Gendle MH, Cory-Slechta DA (2004) Impaired neuropsychological functioning in lead-exposed children. Dev Neuropsychol 26(1):513-540

15. Kordas $\mathrm{K}$ et al (2006) Deficits in cognitive function and achievement in Mexican first-graders with low blood lead concentrations. Environ Res 100(3):371-386

16. Wasserman GA et al (1998) The effect of lead exposure on behavior problems in preschool children. Am J Public Health 88(3):481-486

17. Canfield RL et al (2003) Intellectual impairment in children with blood lead concentrations below 10 microg per deciliter. N Engl J Med 348(16):1517-1526

18. Lanphear BP et al (2005) Low-level environmental lead exposure and children's intellectual function: an international pooled analysis. Environ Health Perspect 113(7):894-899

19. Tellez-Rojo MM et al (2006) Longitudinal associations between blood lead concentrations lower than $10 \mathrm{microg} / \mathrm{dL}$ and neurobehavioral development in environmentally exposed children in Mexico City. Pediatrics 118(2):e323-e330

20. Carlisle JC et al (2009) A blood lead benchmark for assessing risks from childhood lead exposure. J Environ Sci Health A Tox Hazard Subst Environ Eng 44(12):1200-1208

21. Schnaas L et al (2006) Reduced intellectual development in children with prenatal lead exposure. Environ Health Perspect 114(5):791-797

22. Ellis BJ et al (1999) Quality of early family relationships and individual differences in the timing of pubertal maturation in girls: a longitudinal test of an evolutionary model. J Pers Soc Psychol 77(2):387-401

23. Schermerhorn AC et al (2013) Temperament moderates associations between exposure to stress and children's externalizing problems. Child Dev 84(5):1579-1593 